\title{
Arbeitnehmerkapital, Finanzmarktregulierung und Geldpolitik
}

Es ist populär, über die Macht der globalen Finanzmärkte und über schädliche Einflüsse von Hedgefonds und anderen Anlegern auf Löhne und Beschäftigung zu klagen. Dass ein beträchtlicher Teil des an den Finanzmärkten bewegten Kapitals Arbeitnehmern gehört und deren Vertretungen durchaus Möglichkeiten zur Einflussnahme auf Anlagestrategien, Spielregeln und Abstimmungen mit der Geldpolitik bieten könnte, wird hingegen nur selten bedacht. Warum eigentlich?

\section{Der andere Faktor}

Neben der Öffnung der Arbeitsmärkte in Transformations- und Schwellenländern, die das weltweite Angebot an Arbeitskraft Anfang der 1990er Jahre um ein Drittel erhöhte, hat die Globalisierung der Finanzmärkte zu anhaltendem Druck auf Beschäftigung und Arbeitseinkommen in den alten Industrieländern beigetragen. Kapitalanleger und Unternehmen verfügen nunmehr über eine Fülle von Investitionsalternativen, die durch Drohung mit Abwanderung ihre Verhandlungspositionen gegenüber regional gebundenen Arbeitnehmervertretungen stärkt und Einflussnahme auf politische Rahmenbedingungen erlaubt. In hochliquiden Phasen des Aufschwungs an den Finanzmärkten treten in allen Wirtschaftsbereichen , aktive Anleger" auf den Plan, die mit Forderungen nach Orientierung auf kurzfristige Renditeziele etablierte Investitionsmuster und das Tarifgefüge infrage stellen. In aggressiveren Fällen werden die Finanzierungsrisiken des Anteilserwerbs den übernommenen Unternehmen aufgebürdet und Letztere werden zerlegt. Daneben setzt sich auf den Finanzmärkten der Trend fort, Risiken aller Art durch innovative Kontrakte immer weiter aufzuspalten, neu zu bündeln und über Markttransaktionen umzuverteilen. Beide Entwicklungen führen nicht immer zu einzel- und gesamtwirtschaftlichen Effizienzgewinnen, sondern erzeugen auch Plünderungsschäden und Systemrisiken, die durch Finanzmarktregulierungen und Geldpolitik nur unzureichend beherrscht werden. Periodisch wiederkehrende Liquiditätskrisen und Kreditklemmen hinterlassen Bremsspuren auf den Arbeitsmärkten und erzeugen anhal- tende Arbeitslosigkeit und Einkommenseinbußen.

Im Zuge dieser Entwicklungen tendieren die Entgelte für den Produktionsfaktor Arbeit in den meisten Industrieländern zu einer relativen Abnahme gegenüber den Kapitaleinkommen. Gewerkschaften und andere Arbeitnehmervertretungen, die auf nationaler Ebene traditionell ein politischer Machtfaktor sind, verfügen in den neuen transnationalen Unternehmensstrukturen und der supranationalen Stabilitätspolitik der Europäischen Währungsunion über weit weniger Einfluss. In Debatten über Probleme der Finanzierung bestehender Sozialversicherungssysteme wird Arbeitnehmern angeraten, vorsorglich Kapital zu bilden und somit auf ,den anderen Faktor" der volkswirtschaftlichen Verteilungsrechnung als zusätzliche Einkommensquelle zu setzen.

Auch wenn man solche Empfehlungen als zynisch empfinden mag, ist nicht zu leugnen, dass ein beträchtlicher Teil des Kapitals, das auf den Finanzmärkten bewegt und in transnational operierenden Anlageinstitutionen investiert wird, als „Arbeitnehmerkapital“ bezeichnet werden kann. Denn es stammt aus Forderungen und Besitz von Personen, die ihren Lebensunterhalt hauptsächlich mit Einkommen aus abhängiger Beschäftigung verdienen. Kapital aus Arbeitnehmerhand existiert unter anderem in Form von Pensionskassen und -fonds, Versicherungen, Mitarbeiterbeteiligungen, Stiftungen und überbetrieblichen Tariffonds. Das globale Gesamtvolumen lässt sich kaum beziffern, doch einen groben Anhaltspunkt für die Größenordnung des Arbeitnehmerkapitals bietet das aggregierte Finanzvermögen der Pensionsfonds in der OECD (wobei „Pensionsfonds“ hier den Oberbegriff für institutionelle Anleger mit Bezug zur Altersvorsorge bildet). Dieses belief sich im Jahre 2005 auf 87,6\% des
Bruttoinlandsprodukts der OECD und auf knapp 50 \% der Marktkapitalisierung aller börsennotierten Unternehmen in diesen Ländern; seit 1995 war es jährlich im Durchschnitt um ca. $10 \%$ gewachsen (OECD 2004 und 2006, S. 3; World Bank 2007). Ein Teil der Pensionsfonds speist sich zwar aus Beiträgen von Selbstständigen und leitenden Angestellten und ist insoweit kaum als Arbeitnehmerkapital zu betrachten; sein Abzug würde aber vermutlich durch das Arbeitnehmerkapital in anderen Anlagen aufgewogen.

In jedem Falle stellt sich die Frage, ob das vorhandene Arbeitnehmerkapital seinen Eigentümern und deren Vertretungen als Basis für stärkere Mitsprache auf verschiedenen Ebenen dienen kann: auf der einzelwirtschaftlichen Ebene von Unternehmen, auf der sektoralen Ebene der Finanzmarktregulierungen, vielleicht gar auf der gesamtwirtschaftlichen Ebene der Koordination von Zins- und Lohnpolitik. Bei positiven Antworten folgen weitere Fragen: Könnte die kapitalbasierte Mitsprache für die Durchsetzung von Arbeitnehmerinteressen genutzt werden? Gibt es hierbei nicht Konflikte zwischen Lohnund Renditeinteressen? Ist kapitalbasierte Mitsprache überhaupt eine Aufgabe für Gewerkschaften? Oder ist sie für diese gar eine Überlebensnotwendigkeit, um Machtverluste in Tarifpolitik und arbeitsrechtlicher Mitbestimmung auszugleichen?

\footnotetext{
Hans-Michael Trautwein, Dr., Professor für internationale Wirtschaftsbeziehungen an der Carl von Ossietzky Universität Oldenburg. Arbeitsschwerpunkte: Makroökonomische Theorieentwicklung, Monetäre Integration und internationale Finanzmärkte, Transnationalisierung der Produktion. e-mail: michael.trautwein@uni-oldenburg.de
} 
Eine Prüfung der Möglichkeiten kapitalbasierter Mitsprache findet in Gewerkschaftskreisen, zumindest in Deutschland, kaum statt. Die Gründe für diesen blinden Fleck sind vielfältig: Bei Mitarbeiterbeteiligungen sieht man Loyalitätskonflikte und Eigentumsrisiken, die zu den Beschäftigungsrisiken hinzutreten. Investivlohninitiativen werden - nicht immer zu Unrecht - als tarifpolitische Ablenkungsmanöver der Gegenseite abgetan. Bei überbetrieblichen Formen von Arbeitnehmerkapital werden Rollenkonflikte zwischen arbeits- und eigentumsrechtlicher Mitbestimmung befürchtet. Pensionsfonds spielen in Deutschland eine weitaus geringere Rolle als beispielsweise in den Niederlanden, USA, Großbritannien oder Dänemark. Zudem handelt es sich bei Finanzmarkttransaktionen und -regulierungen um eine komplexe Materie, die ein schwer vermittelbares Ausmaß an Expertenwissen erfordert. Sicherlich spielen auch Erinnerungen an diverse Finanzmarktdesaster gemeinwirtschaftlicher Unternehmen eine Rolle; hierzu zählen z. B. in Deutschland und Österreich die spektakulären Verlustgeschäfte der Banken AHBR und BAWAG, die in den Jahren 2005 und 2006 Gewerkschaftsvermögen in großem Stil vernichteten.

Alle diese Bedenken sollten einer systematischen Prüfung der Optionen kapitalbasierter Mitsprache nicht entgegenstehen; sie sind vielmehr auf ihre Triftigkeit abzuklopfen. Der vorliegende Aufsatz liefert einen Beitrag hierzu, indem er aus ökonomischer Sicht (unter Vernachlässigung juristischer Aspekte) die Möglichkeiten und Probleme von kapitalbasierter Mitsprache in internationaler Perspektive auf drei Ebenen skizziert: auf der Mikroebene der einzelnen Unternehmen (Abschnitt 2), auf der Mesoebene der Regulierung von Finanzmärkten (Abschnitt 3) und auf der Makroebene der Koordination von Zins- und Lohnpolitik (Abschnitt 4). Dabei wird vorausgesetzt, dass sich das vorhandene Arbeitnehmerkapital für die Einflussnahme auf allen drei Ebenen organisieren ließe. Wie realitätsnah diese Hypothese ist, lässt sich aufgrund der schlechten Datenlage kaum beurteilen. Ein umfassender internationaler Überblick über Formen und Umfang von Arbeitnehmerkapital, bei dem nach Kontrollrechten und Anlagerestriktionen differenziert wird, liegt (noch) nicht vor. Doch auch in der Beschränkung auf kühne Hypothesen kann die Prüfung der
Mitspracheoptionen mehr sein als ein akademisches Glasperlenspiel. Durch unvoreingenommene und sorgfältige Unterscheidung zwischen dem, was unter bestimmten Umständen Realität werden kann, und dem, was bloße Illusion bleiben muss, kann sie helfen, die Diskussion über ein bislang vernachlässigtes Thema in Gang zu bringen (Trautwein 2006). Mit der Analyse der Optionen wird zudem der Datenbedarf für empirische Studien von Arbeitnehmerkapital und Finanzmarktregulierungen genauer definiert.

\section{Kapitalbasierte Mitsprache auf Unternehmensebene}

Unter dem Aspekt der Mitsprache lassen sich verschiedene Formen von Arbeitnehmerkapital nach den Merkmalen „Anteilseignerschaft", „Kontrollrechte“ „Mitarbeiterbeteiligung" und „Anlagesektor" unterscheiden. Schon in diesen groben Kategorien, bei denen von Unterschieden zwischen nationalen Regelungen und anderen institutionellen Details abstrahiert wird, gibt es eine Vielzahl von Kombinationsmöglichkeiten mit verschiedenen Graden der Mitsprachemöglichkeit.

Ein großer Teil des Arbeitnehmerkapitals wird in Kundenbeziehungen mit Finanzdienstleistern gebildet, $\mathrm{z}$. B. in Lebensversicherungen oder anderen Sparplänen bei kommerziellen Anbietern. Damit sind in der Regel keine Mitspracherechte verbunden. Da Arbeitnehmer hierbei als individuelle Konsumenten auftreten, denen Engagement kaum Ertrag verspricht, lässt sich die Wahrnehmung ihrer Interessen nach der Logik des kollektiven Handelns (Olson 1965) allenfalls in extremen Fällen drohender Verluste organisieren. Von Mitsprache wird man eher dort reden können, wo Arbeitnehmer Anteilseigner sind, also direkt oder indirekt über Fonds Aktien oder andere Anteile besitzen. Auch in diesem Fall gibt es unterschiedliche Ausstattungen mit Stimm- und weiteren Kontrollrechten. Des Weiteren ist danach zu unterscheiden, ob es sich um Anteile an den Unternehmen handelt, in denen die betreffenden Arbeitnehmer beschäftigt sind (z. B. Belegschaftsaktien), oder um Anteile an anderen Unternehmen. Bei Pensions- und Versicherungsfonds werden häufig die Kontrollrechte von den jeweili- gen Belegschaften getrennt, da sie wegen vorgeschriebener oder selbstgewählter Risikodiversifikation Kapital nicht dort anlegen, woher die Anlagegelder stammen. Im Hinblick auf die Einflussnahme auf Finanzmarktregulierungen macht es außerdem einen Unterschied, ob Anteile an Unternehmen im Finanzsektor (Banken, Fondsgesellschaften u.a.) oder in anderen Sektoren gehalten werden.

Kapitalbasierte Mitsprache in Unternehmen wird hauptsächlich durch Mitgliedschaft in Aufsichtsräten ausgeübt. Ihre zentralen Funktionen bestehen im Zugang zu Informationen und in der Kontrolle von Strukturentscheidungen der Unternehmensführung. Bei der Repräsentanz von Arbeitnehmerkapital in Aufsichtsräten (die von der Repräsentanz nach Mitbestimmungs- und Drittelbeteiligungsgesetz zu unterscheiden ist) kann man aus der gewerkschaftsseitigen Kritik an aktuellen Entwicklungen auf Finanz- und Arbeitsmärkten zwei spezifische Zielsetzungen ableiten: Das erste Ziel wäre die Einschränkung von riskantem Verhalten der Unternehmensführung, das Arbeitsplätze gefährden oder die Arbeitsbedingungen nachhaltig beeinträchtigen könnte. Das zweite Ziel beinhaltet die Durchsetzung von Sozialstandards, insbesondere in transnationalen Unternehmen und Wertschöpfungsketten, die auch in Niedriglohnländern operieren. Darüber hinaus können weitere Ziele verfolgt werden. Abzuwägen ist aber jeweils, inwieweit die Ziele miteinander verträglich sind, vor allem im Verhältnis von Arbeitnehmern als Lohnabhängigen und als Rentiers. Ein weiterer Problemkreis ist die Qualifikation und Loyalität der Vertreter von Arbeitnehmerkapital in den Aufsichtsräten.

\subsection{INTERESSENKONFLIKTE}

Der Nutzen der kapitalbasierten Mitsprache für Arbeitnehmer hängt von der Verträglichkeit ihrer Eigentümerinteressen mit anderen Ansprüchen bezüglich Beschäftigung und Arbeitsbedingungen ab. In betriebswirtschaftlichem Neudeutsch spricht man vom Verhältnis der Shareholder und Stakeholder. Ein Grundkonflikt ist nicht von der Hand zu weisen. In Bezug auf Beschäftigung und Qualifikation sind Arbeitnehmer zunächst einmal Stakeholder im Unternehmen. Als Kapitalbesitzer sind sie Shareholder, entweder im selben Unternehmen oder in Fonds, die in andere Unter- 
nehmen investiert haben. In der Regel hat Arbeitnehmerkapital Einkommenserzielung als Primärzweck und ist daher auf Rendite ausgerichtet. Die Erträge speisen sich aus Unternehmensgewinnen, mithin aus der Differenz zwischen Erlösen und Kosten. Da Arbeit einen Kostenfaktor darstellt, bedeutet die Maximierung dieser Differenz im Allgemeinen einen Zielkonflikt zwischen Lohn- und Kapitalertragszuwächsen. Dieser Zusammenhang ist wohlbekannt, und das berüchtigte Schlagwort Shareholder Value weist zunächst nur darauf hin, dass Gewinnmaximierung als Interesse der Anteilseigner auch oberstes Ziel der Unternehmensführung sein sollte.

Die Verwendung desselben Begriffs für aggressiven Anlegeraktivismus deutet zugleich an, dass die gängige Definition des Gewinns als Differenz von Erlösen und Kosten einen Teil des Konflikts verdeckt. Denn die ökonomische Definition des Gewinns stellt auf die Differenz von Erlösen und Opportunitätskosten ab. Es genügt damit nicht, dass eine Investition einen Überschuss über die geleisteten Geldzahlungen erbringt; sie muss auch mindestens so profitabel sein wie der Durchschnitt der alternativ realisierbaren Investitionen. Mit der Globalisierung der Finanzmärkte und der Transnationalisierung der Produktion hat sich diese Gewinndefinition deutlicher durchgesetzt. Auch Pensionsfonds und andere Gesellschaften, in denen Arbeitnehmer Kapital angelegt haben, verfahren danach. Die enorme Zunahme von Investitionsalternativen hat den Druck auf die Arbeitskosten erhöht und Interessenkonflikte zwischen Anteilseignern und Beschäftigten entsprechend verschärft. Nicht selten werden die Konflikte mit nationalistischen Argumentationsmustern zu Invasionen stilisiert, etwa wenn Hedgefonds, in denen auch ausländische Pensionsfonds investiert sind, Unternehmen übernehmen. Die Nationalität der Anleger ändert freilich wenig an der Sache. Die Konflikte und ihre Folgen für die Beschäftigten in den betroffenen Unternehmen wie auch für die kapitalanlegenden Arbeitnehmer sind meist nur im spezifischen Kontext bestimmbar, aber nicht immer unvermeidlich. Es folgen einige Vorüberlegungen zur Entwicklung eines analytischen Rahmens, in dem Bereiche der Übereinstimmung der Interessen identifiziert und Regeln für die Bewältigung von Shareholder/Stakeholder-Konflikten definiert werden können.

\subsection{KONFLIKTBEWÄLTIGUNG}

Zumindest ein Teil der Konflikte zwischen gewerkschaftlich vertretenen Arbeitnehmern und institutionellem Arbeitnehmerkapital kann durch Verhandlungen entschärft werden. Es ist schwierig, allgemeine und dennoch aussagekräftige Regeln für solche Dialoge zu beschreiben, da sich die Kommunikationskanäle je nach Organisationsstruktur der Gewerkschaften von Branche zu Branche und Land zu Land stark unterscheiden. Die Etablierung weithin anerkannter Mediatoren und Mediationsregeln, wie sie in arbeitsrechtlichen und handelspolitischen Zusammenhängen verbreitet sind, könnte helfen. Solche Vermittlungsinstanzen sind besonders relevant bei transnationalen Investitionen von Arbeitnehmerkapital. Wenn beispielsweise die Belegschaft eines deutschen Unternehmens in Konflikt mit einem britischen Pensionsfonds gerät, der am Unternehmen beteiligt ist, wäre es hilfreich, wenn die Parteien ein supranationales Schiedsgericht oder ein internationales Mediatorennetzwerk in Anspruch nehmen könnten.

\section{3 ÜBEREINSTIMMUNG VON INTERESSEN}

Der Grundkonflikt von Rendite- und Lohnzielen schließt nicht aus, dass die Vertreter von Arbeitnehmerkapital und Belegschaften der betreffenden Unternehmen gemeinsame Interessen verfolgen. Es gibt mindestens drei Bereiche, in denen dies der Fall sein dürfte: die Abwehr von „räuberischen Investoren“, die Unterbindung übermäßig riskanten Verhaltens der Unternehmensführung sowie die Zahlung von Effizienzlöhnen.

Feindliche Übernahmen von Unternehmen sind nicht per se zum Schaden von deren Belegschaften, denn daraus folgende Restrukturierungen können auf lange Sicht durchaus zu mehr Beschäftigung und Einkommen führen als der Status quo (zur Empirie siehe z. B. Croci 2005). Im Falle eines sogenannten LBO (Leveraged Buy-Out) ist der Erwerb jedoch in hohem Maße zulasten der übernommenen Unternehmen schuldenfinanziert und führt in aller Regel zur Zerschlagung und Vermögensentnahme (Asset Stripping) - mit allen Risiken für die Belegschaft, einschließlich der Pensionsrückstellungen (sofern diese nicht in unabhängigen Kassen oder Fonds gebildet sind). Bei gehäuftem Auftreten von LBO steigen auch die Preisrisiken auf Märkten für Unternehmensanteile. Pensionsfonds und andere Verwalter von Arbeitnehmerkapital verfolgen in der Regel langfristig orientierte Anlagestrategien. Eine allgemein wahrnehmbare Zunahme der Volatilität der Anteilspreise ist ihrer Attraktivität eher abträglich. Daher kann es in LBO-bedrohten Unternehmen Koalitionen zur Abwehr feindlicher Übernahmen geben, insbesondere dort, wo Arbeitnehmerkapital bereits investiert war, und dort, wo durch Erwerb oder Aufstockung von Anteilen eigene Anlagestrategien deutlich signalisiert werden können.

„Übermäßig“ riskantes Verhalten des Managements, oft auch veranlasst durch Forderungen ,aktiver Anleger" mit Minderheitsbeteiligung, hat ähnliche Konsequenzen wie ein LBO. Es ist zwar schwierig, im Vorhinein übermäßige von normalen Risiken zu unterscheiden. Häufig lässt sich jedoch erkennen, wenn die Unternehmensführung ohne entsprechendes eigenes Risiko zulasten der Belegschaft und des Arbeitnehmerkapitals handelt. In solchen Fällen eines Moral Hazard (moralisch riskantes Verhalten) liegt Kooperation naturgemäß im Interesse der Vertreter von Arbeitnehmerkapital und Belegschaft.

Der Begriff der Effizienzlöhne beschreibt Fälle, in denen Arbeitgeber die Produktivität steigern und Stückkosten senken können, indem sie die Motivation der Mitarbeiter durch Lohnzulagen und Verbesserung der Arbeitsbedingungen erhöhen. Diese Mechanismen sind oft an Strategien der langfristigen Mitarbeiterbindung und Investitionen in entsprechende Ausbildung gekoppelt. Wenn mit Arbeitnehmerkapital im Durchschnitt langfristige Anlagestrategien verfolgt werden, in denen die höheren Festlegungsrisiken durch stabile Erträge ausgeglichen werden, steigt gegenüber dem Durchschnitt der Kapitalanleger die Bereitschaft, Effizienzlohnargumenten zu folgen. Entsprechende Initiativen in führenden Unternehmen können auf ganze Industrien ausstrahlen.

Auf der Basis dieser und weiterer Überlegungen könnten Kooperationsstrategien entwickelt werden, die an die spezifischen Rahmenbedingungen der Corporate Governance im jeweiligen Land und in transnationalen Strukturen angepasst sind und auf Komplementarität zu Strukturen arbeitsrechtlicher Mitbestimmung achten, wo diese vorhanden sind. Die wachsende 
Literatur zur politischen Ökonomie dieser Rahmenbedingungen bietet vielfältige Hilfestellungen (Pagano/Volpin 2001 und 2005; Jacobi et al. 2007; Graz/Nölke 2008).

\subsection{VERTRETUNGSPROBLEME}

Für die Verwaltung des Fondskapitals, das mit Arbeitnehmergeldern gebildet wird, wie auch für die Ausübung der Kontrollrechte, die mit Arbeitnehmerkapital verbunden sind, werden hinreichend qualifizierte und loyale Manager benötigt. Wie der Niedergang der Gemeinwirtschaft in Deutschland in den 1980er Jahren zeigt, verschärfen sich die üblichen PrincipalAgent-Probleme der Kontrolle von Managern durch die Anteilseigner im gewerkschaftsnahen Bereich allerdings, wenn die Zielsetzungen der Anteilseigner aufgrund von Konflikten der Renditeorientierung mit gewerkschaftlichen Interessen diffus bleiben (Hassel 2003). Dies verschafft den Fondsmanagern und Aufsichtsratsmitgliedern Spielräume für eigenständiges Handeln, das nicht unbedingt im Interesse ihrer Auftraggeber ist und gerade an Finanzmärkten durch Fehlspekulationen „auf fremde Rechnung" zu gravierenden Vermögensverlusten führen kann.

Im Bereich der Rekrutierung von Aufsichtsratsvertretern gibt es ebenfalls Principal-Agent-Probleme, ähnlich wie bei der arbeitsrechtlichen Mitbestimmung. Hier besteht die Gefahr, dass sich hohe Loyalität mit geringer Professionalität paart und umgekehrt. Aufsichtsratsvertretern mit gewerkschaftlichem Hintergrund fehlt nicht selten das Wissen, das zur Beurteilung und Mitgestaltung zunehmend komplexer Finanzierungs- und Führungsstrukturen in transnationalen Unternehmen notwendig ist. Aufsichtsratsvertreter, die aus Finanzkreisen oder anderen Expertenzirkeln stammen, spielen hingegen häufig nach anderen Regeln und mitunter ihr eigenes Spiel. Entscheidend für den Erfolg von Institutionen, die Arbeitnehmerkapital mit entsprechendem Profil vertreten, ist somit auch die Frage, ob hinreichend anreizverträgliche Systeme der Rekrutierung, Ausbildung und Vergütung von Managern entwickelt werden können.

\section{Kapitalbasierte Mitsprache bei Finanzmarkt- regulierungen}

Die zweite Ebene, auf der kapitalbasierte Mitsprache von Arbeitnehmern zum Tragen kommen könnte, ist Repräsentanz in der Finanzmarktregulierung. Hier ist zu beachten, dass es trotz zunehmender Marktintegration und Harmonisierung von Vorschriften in der Europäischen Union eine Vielfalt von Organisationsformen gibt, die von staatlichen Megaaufsichtsbehörden bis zur Selbstregulierung von Teilbranchen reicht (Goodhart et al. 1998; Leading International Law Firms 2005).

Als Kapitalanlagegesellschaften oder andere Finanzdienstleister sind Verwalter von Arbeitnehmerkapital Regulierungsobjekte. Als Großkunden von Banken und anderen Finanzdienstleistern sind sie Nutznießer der Regulierungen, sofern diese ihre Hauptaufgaben - Anlegerschutz und Sicherung der Stabilität des Finanzsystems - erfüllen. In beiden Rollen könnten Verwalter von Arbeitnehmerkapital bei Bündelung ihrer Kräfte Vertreter in Gremien von Regulierern entsenden. Ein Blick auf Aufsichtsgremien in den drei größten Volkswirtschaften Europas zeigt exemplarisch, dass solche Überlegungen nicht ganz abwegig sind. In mehreren Beiräten der deutschen Bundesanstalt für Finanzdienstleistungsaufsicht (BAFin) sind Arbeitnehmervertreter qua Statut Mitglieder; Repräsentanz von Arbeitnehmerkapital im Verwaltungsrat wäre prinzipiell möglich. In der französischen Autorité des Marchés Financiers hat die Vertretung der Belegschaftsaktionäre sogar einen festen Sitz im Verwaltungsrat. In der britischen Financial Supervisory Authority gibt es zwar keine satzungsmäßige Vertretung von Arbeitnehmern, aber Verwaltungsratsmitglieder mit gewerkschaftlichem (oder vergleichbarem) Hintergrund. In allen bestehenden Organisationsformen der Regulierung ist die Gremienmitgliedschaft von Vertretern der Finanzdienstleister vorgesehen. Bei entsprechendem Gewicht könnten Verwalter von Arbeitnehmerkapital solche Vertretungen übernehmen.

Wie auf der Unternehmensebene bestehen die Hauptfunktionen regulatorischer Mitsprache von Arbeitnehmerkapital im Zugang zu Informationen und in der Mitwirkung an Entscheidungen hier über die Gestaltung von Regeln und Überwachung ihrer Einhaltung. Die Zielsetzungen lassen sich ebenfalls als „Beschränkung von übermäßigen Risiken “ und „Durchsetzung von Mindeststandards" umschreiben. Mit ihrer Präzisierung beginnen allerdings die Probleme. Analog zum Grundkonflikt von Lohnund Renditeinteressen (siehe Abschnitt 2) treten Gegensätze im Hinblick auf die Intensität der Regulierungen auf: Als Finanzdienstleister präferieren Arbeitnehmerkapitalverwalter Freizügigkeit, während sie als Kunden und Vertreter von Kleinanlegern und Gewerkschaften an strengeren Regulierungen interessiert sind. Zudem ist auch auf der Regulierungsebene mit Vertretungsproblemen zu rechnen.

\section{1 ÜBER- UND UNTERREGULIERUNG}

Im Hinblick auf die regulatorische Mitsprache von Arbeitnehmerkapitalvertretern stellen sich zwei Fragen: Gibt es besondere Auswirkungen von Finanztransaktionen auf Arbeitnehmer, die spezielle Finanzmarktregulierungen erfordern? Wenn ja: Wären solche speziellen Regulierungen mit den allgemeinen Zielen des Anlegerschutzes und der Stabilität des Finanzsystems vereinbar? Man kann argumentieren, dass Lohnabhängige ein besonderes Interesse an der Einschränkung von Finanztransaktionen haben, die durch Vernichtung von Arbeitsplätzen und Arbeitseinkommen soziale Kosten verursachen - wie z. B. ein Teil der bereits angesprochenen LBO. Solche Regulierungen würden zwar über Belange des Anlegerschutzes und der Finanzsystemstabilität hinausgehen, wären aber gemeinwohlverträglich, wenn sie gesamtwirtschaftliche Beschäftigungs- und Einkommensverluste verhindern, ohne wachstumsfördernden Strukturwandel zu behindern. Da Finanzmarktregulierungen im politischen Kräftespiel von Parteien, Lobbies der regulierten Branchen und anderen Akteuren entstehen, gäbe es hier eine besondere Rolle für regulatorische Mitsprache von Arbeitnehmerkapital, auf die unten noch eingegangen wird.

Allerdings ist es schwer, zwischen sozialschädlichen und sozialverträglichen Finanzierungsformen so zu unterscheiden, dass trennscharf reguliert werden könnte. Zunächst müsste dargelegt werden, in welcher Hinsicht die bestehenden und geplan- 
ten Regulierungen (einschließlich Basel II ${ }^{1}$ und der EU-Finanzmarktdirektive, Mifid) unzureichend sind. Auch wenn Defizite ausgemacht werden, besteht bei weitergehenden Vorschriften die Gefahr einer Überregulierung, die Negativauslese erzeugt. Finanzierungsrestriktionen könnten sich als ineffizient erweisen, indem sie die Kosten solider Projekte so erhöhen, dass die Investitionen in Realkapital und Arbeitsplätze zurückgehen. Zugleich könnten sie ineffektiv sein, da die eigentlichen Adressaten der Restriktionen auf globalen Finanzmärkten über Ausweichmöglichkeiten verfügen und Regulierungsarbitrage sogar einen eigenen Geschäftszweig bildet: Man verlagert die Geschäfte bei Bedarf an weniger stark überwachte Finanzplätze und in weniger streng regulierte Marktsegmente (Goodhart et al. 1998, S. 61ff.).

Andererseits ist nicht auszuschließen, dass Verwalter von Arbeitnehmerkapital ein Eigeninteresse an "Unterregulierung“ entwickeln. Pensionsfonds und Lebensversicherer sind aufgrund ihres treuhänderischen Charakters besonders strengen Vorschriften unterworfen. Gleichzeitig konkurrieren sie mit Finanzdienstleistern, die durch Regulierungsarbitrage zu geringeren Kosten operieren. Auch andere Verwalter von Arbeitnehmerkapital, die hochrentable Anlagen suchen und dabei auf Beteiligungen an Hedgefonds, LBO und anderen risikofreudigen Projekten zurückgreifen, sind an Beschränkungen solcher Geschäfte nicht unbedingt interessiert. Regulatorische Mitsprache von Arbeitnehmerkapital könnte aufgrund von Renditeinteressen auch zur Aufweichung bestehender Standards beitragen.

Diskussionen über Über- und Unterregulierung setzen voraus, dass sich ein optimales Maß an Regulierung bestimmen lässt. Jenseits stark reduzierter Modelle ist diese Übung keineswegs trivial, wie z. B. die Diskussionen um das Basel II-Abkommen als Richtmarke für Finanzmarktregulierungen zeigen. Basel II wird vielfach als ein Regime kritisiert, das gleichzeitig Unterregulierung und Überregulierung beinhaltet, indem es unkontrollierte Kreditbewertungsagenturen stärkt, die Kostenbelastung kleinerer Banken und Unternehmen überproportional erhöht und durch das Risikobewertungsmodell die Prozyklizität der Kreditmärkte verstärkt (Danielsson et al. 2001; Redak 2006; s. auch Springler in diesem Heft). Der Entstehungsprozess des Basel II-Abkommens gibt auch Hinweise darauf, welche Vertretungsprobleme bei regulatorischer Mitsprache von Arbeitnehmerkapital zu erwarten sind.

\subsection{GEGEN AUSGRENZUNG}

Die Principal-Agent-Problematik der Regulierungsbeteiligung von Arbeitnehmerkapital ist ähnlicher Natur wie auf der Unternehmensebene, hat aber eine zusätzliche Dimension. Die Repräsentation von Arbeitnehmerkapital in Regulierungsgremien erfordert Akteure, die sowohl ihre Arbeitnehmerklientel als auch die jeweilige Finanzbranche und schließlich das Allgemeinwohl glaubwürdig vertreten können. Diese Aufgabe mag auf den ersten Blick unmöglich erscheinen, kann aber unter bestimmten Bedingungen durchaus erfüllt werden.

Institutionen der Finanzmarktregulierung sind keine neutralen Zonen, in denen die Definition und Durchsetzung des Allgemeinwohls von vornherein höchste Priorität hat. Sie sind politische Märkte, in denen die regulierten Branchen mit ihrer Finanzkraft und ihren Wissensvorsprüngen Lobbyarbeit betreiben, um Regulierungen in ihrem Sinne zu beeinflussen. Zwar gibt es, wie gezeigt, satzungsgemäße und informelle Vertretung von Arbeitnehmerinteressen in einigen Aufsichtsgremien. Dabei handelt es sich aber meist um Überbleibsel aus korporatistischen Traditionen; die gegenwärtigen Trends der Finanzmarktregulierung scheinen eher auf eine Ausgrenzung von Arbeitnehmern und anderen Interessengruppen hinzudeuten (Mooslechner et al. 2006). Grenzüberschreitende Finanzmärkte (insbesondere im Derivatehandel) werden von nationalen Regulierungsbehörden bestenfalls partiell kontrolliert. Es haben sich Geflechte von transnationaler privater Selbstregulierung und lokalbehördlicher Regulierung herausgebildet, die zum Teil wenig transparent sind und über deren Krisenfestigkeit Unsicherheit besteht. In den großen Finanzzentren setzen zuständige Regierungen vielfach auf die Selbstverwaltung von Branchen, die wegen hoher Innovationsdynamik oder oligopolistischer Strukturen schwer von außen zu regulieren sind, aber starke Eigeninteressen an Koordination der Konkurrenz aufweisen. In internationalen Initiativen zur Harmonisierung von Finanzmarktstandards, allen voran das Basel II-Abkommen, sind die regulierten Branchen am Diskussionsprozess regelmäßig und maßgeblich beteiligt, während andere als Kunden und dritte betroffene Gruppen von den Regulierungen ausgegrenzt bleiben.

In den neuen Strukturen der trans- und internationalen Finanzmarktregulierung finden Arbeitnehmerstimmen nicht leicht Gehör. Auf der Basis von eigener Finanzkraft und Expertise dürfte regulatorische Mitsprache aber eher zu erreichen sein als über Initiativen zur Repräsentation von Verbandsinteressen nach korporatistischem Muster. Insbesondere nach Krisen an Finanzmärkten, die für gewöhnlich Diskussionen über Regulierungsversagen auslösen, könnte die Einbindung von Arbeitnehmerkapital als gemeinwohlförderliche Gegenlobby politische Schubkraft erhalten. Denn es lässt sich argumentieren, dass Institutionen, die mit der Verwaltung von Arbeitnehmerkapital sowohl den Schutz von Kleinanlegern als auch spezifische Risiken der Lohnabhängigkeit (und damit der sozialen Sicherungssysteme) im Blick haben, das Allgemeininteresse an Systemstabilität besser repräsentieren als andere Finanzdienstleister.

\section{Mitsprache in der Stabilitätspolitik}

In der Literatur über Finanzmarktregulierungen werden feinsinnige Unterscheidungen zwischen spezifischen und systemischen Risiken getroffen, wobei Erstere sich auf Mängel an Können, Sorgfalt und gutem Willen in einzelnen Unternehmen beziehen und Letztere auf die wechselseitige Abhängigkeit der Akteure in Finanzsystemen. Leider nimmt die Realität auf solche Unterscheidungen wenig Rücksicht. Herdenverhalten gilt im Vorhinein meist als rational, ja sogar als risikoavers, weil man nicht gegen den Markt wettet. Dennoch führt es regelmäßig zur Verwandlung von spezifischen Risiken, die im Prinzip versicherbar und regulierbar sind, in Systemrisiken, die dies nicht sind. Wenn die Stabilität des Finanzsystems gefährdet ist, können „normale“ Regulierungen (z. B. Eigenkapitalvorschriften für Banken) die Si-

\footnotetext{
1 „Basel II" steht für die Neuregelung der Kreditvergabe und des Bankenaufsichtsrechts. Vgl. auch Spring/er in diesem Heft.
} 
tuation noch verschlimmern (durch Verschärfung der Kreditklemme), sodass die zeitweilige Außerkraftsetzung von Vorschriften in Kombination mit Liquiditätshilfen der Zentralbank oft den letzten Ausweg aus einer Krise bildet. Solche Kombinationen erfordern die Kooperation der Regulierer mit den Zentralbanken, und zwar über die Regulierung von Geschäftsbanken (in etlichen Ländern teilweise bei der Zentralbank angesiedelt) hinaus in allen Segmenten des Finanzsystems (Goodhart et al. 1998, Kap. 3, 7 und 9; Stiglitz/ Greenwald 2003, Kap. 9). Spätestens seit der Asienkrise 1997, die weltweit zur Verpflichtung halbjährlicher Berichterstattung über die Stabilität des Finanzsystems geführt hat, findet solche Kooperation präventiv auch außerhalb von Krisenzeiten statt.

\subsection{VERLAGERUNG DER MAKRO- ÖKONOMISCHEN KOORDINATION}

Wenn sich Mitsprache von Arbeitnehmerkapital bei Finanzmarktregulierungen durchsetzen lässt und in Zusammenarbeit mit den Gewerkschaften stattfindet, könnte sie den Gewerkschaften einen zusätzlichen Kanal der stabilitätspolitischen Kommunikation mit Zentralbanken eröffnen. Dies wäre insbesondere auf der supranationalen Ebene der europäischen Geldpolitik von Bedeutung. Denn in der Koordination von makroökonomisch relevanten Entscheidungen in Europa haben die Gewerkschaften an Bedeutung eingebüßt. Das lange Jahre signalgebende Spiel zwischen der Tarifpolitik deutscher Gewerkschaften und der Zinspolitik der Bundesbank ist vom Zinsstrukturspiel zwischen der Europäischen Zentralbank (EZB) und den Finanzmärkten abgelöst worden. Diese Verlagerung war nicht nur durch den Wechsel zu supranationaler Geldpolitik bedingt, sondern auch durch die stärkere Finanzmarktorientierung der Investitionspolitik der Unternehmen und die enorme Zunahme des grenzüberschreitenden Kapitalverkehrs. Damit hat sich auch die Bedeutung der oben angesprochenen Kooperation von Finanzmarktregulierern und der EZB erhöht.

Die Auswirkungen von Finanzmarktentwicklungen und Zinspolitik auf die Arbeitsmärkte sind derzeit kein Gegenstand einer verbindlichen Kommunikation, in der sich die EZB verpflichtet sehen würde, Rechenschaft über ihr Handeln abzulegen.
Gleiches gilt für andere Zentralbanken in der OECD - mit partiellen Ausnahmen in den USA und in Großbritannien, zwei der Länder, in denen die Bildung von Arbeitnehmerkapital am stärksten fortgeschritten ist. Das makropolitische Ziel der regulatorischen Mitsprache von Arbeitnehmerkapital bestünde in der Thematisierung der Interaktion von Finanz- und Arbeitsmärkten und entsprechender kommunikativer Unterstützung der Koordination von Geldund Lohnpolitik.

\subsection{VERMÖGENSPREISINFLATION UND ZINSPOLITIK}

Ein zentrales Themengebiet für die Kommunikationsförderung durch Arbeitnehmerkapital wäre die Instabilität der Vermögenspreise, die von der Interaktion von Finanzmärkten und Geldpolitik ausgeht und sich auf Arbeitsmärkte auswirkt. In den 1970er und 1980er Jahren waren die inflationären Impulse, auf die Zentralbanken mit Zinssteigerungen reagierten, vornehmlich mit Preis-Lohn-Spiralen auf Güterund Arbeitsmärkten verbunden. Die folgenden Rezessionen wurden von Politikern und Ökonomen als notwendige Kosten einer glaubwürdigen Geldwertstabilisierung propagiert, die trotz kurzfristig erhöhter Arbeitslosigkeit auch den Arbeitnehmern zugute kommen würde. Nach herrschender Meinung bildete die Geldpolitik der deutschen Bundesbank ein wachstumsförderndes Korrektiv von Schwankungstendenzen der Preise und Löhne, die im Wesentlichen einer verfehlten Tarifpolitik der Sozialpartner zuzuschreiben waren. Spätestens seit den 1990er Jahren gehen die inflationären Impulse jedoch deutlich stärker von den Finanzmärkten aus; auch die kräftigen Rohstoffpreissteigerungen der jüngsten Zeit wurden durch Termingeschäfte und andere Transaktionen in Spekulation auf eine langfristig steigende Güternachfrage ausgelöst und nicht durch einen realen Nachfrageanstieg. Die Lohnentwicklung ist weltweit hinter den Preisen von Finanzaktiva und anderen Vermögenstiteln zurückgeblieben. Vermögenspreise reagieren zugleich sehr sensibel auf geldpolitische Maßnahmen und spielen in der Transmission der Geldpolitik nunmehr eine dominierende Rolle (Bank for International Settlements 1998; Bernanke 2002).

Aufgrund der hohen Volatilität der Vermögenspreise und ihres deutlichen Vorlaufs vor den realen Konjunkturschwan- kungen tendiert auch die herrschende Meinung immer stärker zu der Ansicht, dass die Vermögenspreisbildung an den Finanzmärkten wesentlich zur Instabilität von Güterpreisniveaus, Finanzsystemen und realwirtschaftlichen Wachstumsraten beiträgt. Zwar wird von den Zentralbanken derzeit eine offene Orientierung der Zinspolitik an der Vermögenspreisinflation abgelehnt. Aber die Erfahrungen seit dem Platzen der Blase der „neuen Ökonomie“ im Frühjahr 2000 deuten darauf, dass ein Abwarten der Übertragung von Inflationsimpulsen von Finanzmärkten auf Güterund Arbeitsmärkte Zinsreaktionen erfordert, die ihrerseits alle drei Marktsysteme destabilisieren können. Wenn im Gegenzug Finanz- und Wirtschaftskrisen durch großzügige Liquiditätshilfen der Zentralbanken abgewendet werden (wie im Jahr 2000 vor allem in den USA geschehen), führt reichliche Liquiditätsversorgung bei niedrigen Zinsen zu „moralisch riskantem Verhalten" (Moral Hazard) und spekulativen Blasen an den Finanzmärkten. Sie verstärkt auch den in Abschnitt 2 angesprochenen Anlegeraktivismus auf der Suche nach höheren Renditen. Mit ihrem Eigenanteil an der Vermögenspreisinflation erweist sich die Geldpolitik weniger als Korrektiv denn als Teil eines destabilisierenden Mechanismus. Es ist daher kein Zufall, wenn Zentralbanker in diesem Dilemma auf die Notwendigkeit von restriktiverer Regulierung und stärkerer Kooperation der Geldpolitik mit den Regulierern hinweisen (exemplarisch: Bernanke 2002).

\subsection{ZINS- UND LOHNPOLITIK IN EUROPA}

In der Europäischen Währungsunion kommt zu dieser Problematik noch ein lohnpolitischer Aspekt hinzu, der das Gewicht der Vertretung von Arbeitnehmerkapital in der Finanzmarktregulierung und im Dialog mit der EZB erhöhen könnte. Die einheitliche Geldpolitik führt durch die weitgehende Eliminierung von Differenzialen in den Nominalzinsen dazu, dass selbst geringe Unterschiede in den Inflationsraten der Mitgliedsländer bedeutsame Differenzen in den Realzinsniveaus erzeugen. In Ländern mit höherer Inflation besteht aufgrund niedrigerer Realzinsen die Gefahr von sich selbst verstärkenden Erhöhungen der Vermögenspreise, der Güterpreise und schließlich der Nominallöhne (Beispiele bieten Spanien und Irland 
in der Anfangsphase der Währungsunion). Zunächst gehen solche kumulativen Prozesse mit hohen realwirtschaftlichen Wachstumsraten und einer Verringerung der Arbeitslosigkeit einher. Die zunehmenden Handelsdefizite gegenüber anderen Ländern der Eurozone mit niedrigerer Inflation führen innerhalb der Währungsunion nicht direkt zu Zahlungsbilanzrestriktionen, sodass spekulative Blasen länger anhalten und größer werden können. Ihr Platzen kann sich hingegen im Rahmen der Währungsunion stärker negativ auf Realinvestitionen und Beschäftigung auswirken, da Wechselkursanpassungen ausgeschlossen sind.

Solche und weitere mögliche Konstellationen verdeutlichen, dass im Rahmen der Währungsunion große Anpassungslasten auf der Lohnpolitik liegen. Ihre Bewältigung erfordert in vielen Fällen die Koordination gewerkschaftlicher Tarifpolitik. Diese ist aber, abgesehen von allen institutionellen Problemen gewerkschaftlicher $\mathrm{Zu}$ sammenarbeit in Europa (Jacobi et al. 2007), bei Inflations- und Wachstumsdifferenzen schwer zu erreichen, weil sie Lohnzurückhaltung in den „überhitzten “ Ökonomien erfordert. Vertretungen von Arbeitnehmerkapital könnten hier eine vermittelnde Rol- le in zweierlei Hinsicht spielen. Sie könnten durch regulatorische Mitsprache die EZB und ihre Mitgliedsbanken zum makroökonomischen Dialog mit den Gewerkschaften anregen, mit dem Ziel, Stabilitätsprobleme durch Kombinationen koordinierter Lohnpolitik mit lokal differenzierenden Maßnahmen der Geldpolitik und Finanzmarktregulierung zu entschärfen. Sie könnten zudem den Tarifpartnern in den überhitzten Ökonomien die weitere Bildung von Arbeitnehmerkapital (in möglichst wertbeständigen Formen) als optionale Kompensation für Lohnzurückhaltung anbieten. Damit würde sich der Kreis schließen. Denn es gäbe dann auch eine makroökonomische (Teil-)Antwort auf die Ausgangsfrage, in welchen Bereichen die Stellung von Arbeitnehmern als Anteilseigner mit ihren Interessen im Hinblick auf Löhne und Arbeitsbedingungen vereinbar ist und diese auch befördern kann.

\section{Schlussbemerkung}

Der vorliegende Aufsatz hat stark spekulativen Charakter, weil er voraussetzt, dass das vorhandene Arbeitnehmerkapital ausreichen kann, um Repräsentanz auf den drei diskutierten Ebenen zu erreichen. Gegenwärtig kann man diese Thematik aber kaum anders erörtern, denn empirische Untersuchungen, die ein Urteil über die Plausibilität dieser Arbeitshypothese erlauben, stehen noch aus. Schon die rein hypothetische - und hier auch nur holzschnittartige - Diskussion der Optionen kapitalbasierter Mitsprache ist jedoch aufschlussreich. Sie zeigt, dass es selbst bei erfolgreicher Durchsetzung beträchtliche Probleme der Zielbestimmung, Konfliktbewältigung und Vertretungseffizienz gibt, die den Einfluss von Arbeitnehmerkapital beschränken. Gleichwohl sind die Probleme nicht von vornherein unlösbar. Auch wenn der Weg lang und schmal ist, besteht die Chance, dass Arbeitnehmervertretungen auf dem komplementären Weg der kapitalbasierten Mitsprache zumindest einen kleinen Teil des Einflusses zurückgewinnen, den sie im Zuge der Finanzmarktglobalisierung verloren haben. Aus ökonomischer Sicht wäre es nicht rational, wenn sie die Gangbarkeit dieses Weges nicht wenigstens prüfen würden. 
Bank for International Settlements (1998): Asset Prices and Monetary Stability: Four Views, Basel

Bernanke, B. (2002): Asset Price "Bubbles" and Monetary Stability, Washington, http://www.federalreserve.gov/BoardDocs/speeches/2002/ 20021015/default.htm

Croci, E. (2005): Corporate Raiders, performance and governance in Europe, erscheint in European Financial Management 13 (November 2007), http://papers.ssrn.com/sol3/papers.cfm?abstract_id=489505 Danielsson, J./Embrechts, P./Goodhart, C. A. E./Keating, C./Muennich, F./Renault, O./Shin, S. H. (2001): An Academic Response to Basel II, London School of Economics Financial Markets Group, Special Paper 130, http://www.bis.org/bcbs/ca/fmg.pdf

Goodhart, C. A. E./Hartmann, P./Llewellyn, D./Rojas-Suárez, L./Weisbrod, S. (1998): Financial Regulation: Why, how and where now? London

Graz, J.-C./Nölke, A. (2008) (Hrsg.): Transnational Private Governance and its Limits, Abingdon

Hassel, A. (2003): Wer beherrschte die Gemeinwirtschaft? Unternehmenskontrolle in politischen Unternehmen, in: Streeck, W./Höpner, M. (Hrsg.): Alle Macht dem Markt? Fallstudien zur Abwicklung der Deutschland AG, Frankfurt/New York, S. 93-117

Jacobi, O./Jepsen, M./Keller, B./Weiss, M. (2007) (Hrsg.): Social Embedding and the Integration of Markets. An Opportunity for Transnational Trade Union Action or an Impossible Task? Düsseldorf Leading International Law Firms (2005): Financial Services Regulation in Europe, Oxford
Mooslechner, P./Schuberth, H./Weber, B. (2006) (Hrsg.): The Political Economy of Financial Market Regulation. The Dynamics of Inclusion and Exclusion, Cheltenham

OECD (2004): Global Pension Statistics Projects: Measuring the Size of Private Pensions with an International Perspective, Paris OECD (2006): Pension Markets in Focus - Issue 3 (October), Paris Olson, M. (1965): The Logic of Collective Action: Public Goods and the Theory of Groups, Cambridge, Mass.

Pagano, M./Volpin, P. (2001): The political economy of finance, in: Oxford Review of Economic Policy 17, S. 502-519

Pagano, M./Volpin, P. (2005): The political economy of corporate governance, in: American Economic Review 95, S. 1005-1030

Redak, V. (2006): Risks, ratings and regulation: toward a reorganisation of credit via Basel II?, in: Mooslechner, P./Schuberth, H./Weber, B. (Hrsg.): The Political Economy of Financial Market Regulation. The Dynamics of Inclusion and Exclusion, Cheltenham, S. 191-213

Stiglitz, J./Greenwald, B. (2003): Towards a New Paradigm in Monetary Economics, Cambridge

Trautwein, Hans-Michael (2006): Employee participation in financial regulation and macroeconomic policies, in: Bercusson, Brian (Hrsg.): Paths to Progress. Mapping Innovation on Information, Consultation and Participation for Employee Involvement in Corporate Governance, Brüssel, S. 126-140 World Bank (2007): Data \& Statistics - Finance, Washington, http://web. worldbank.org/WBSITE/EXTERNAL/DATASTATISTICS/ 